\title{
EchoGéo
}

$47 \mid 2019$

Nouvelles géographies de la collecte

\section{Nouvelles géographies de la collecte : Perspectives croisées sur les « cueilleurs » contemporains}

Introduction

Lucie Dejouhanet et Rémi de Bercegol

\section{OpenEdition}

Journals

Édition électronique

URL : https://journals.openedition.org/echogeo/17255

DOI : 10.4000/echogeo.17255

ISSN : 1963-1197

Éditeur

Pôle de recherche pour l'organisation et la diffusion de l'information géographique (CNRS UMR 8586)

Référence électronique

Lucie Dejouhanet et Rémi de Bercegol, « Nouvelles géographies de la collecte : Perspectives croisées sur les « cueilleurs » contemporains », EchoGéo [En ligne], 47 | 2019, mis en ligne le 21 avril 2019, consulté le 11 août 2021. URL : http://journals.openedition.org/echogeo/17255 ; DOI : https://doi.org/ 10.4000/echogeo. 17255

Ce document a été généré automatiquement le 11 août 2021.

EchoGéo est mis à disposition selon les termes de la licence Creative Commons Attribution - Pas d'Utilisation Commerciale - Pas de Modification 4.0 International (CC BY-NC-ND) 


\section{Nouvelles géographies de la collecte : Perspectives croisées sur les « cueilleurs » contemporains}

Introduction

Lucie Dejouhanet et Rémi de Bercegol

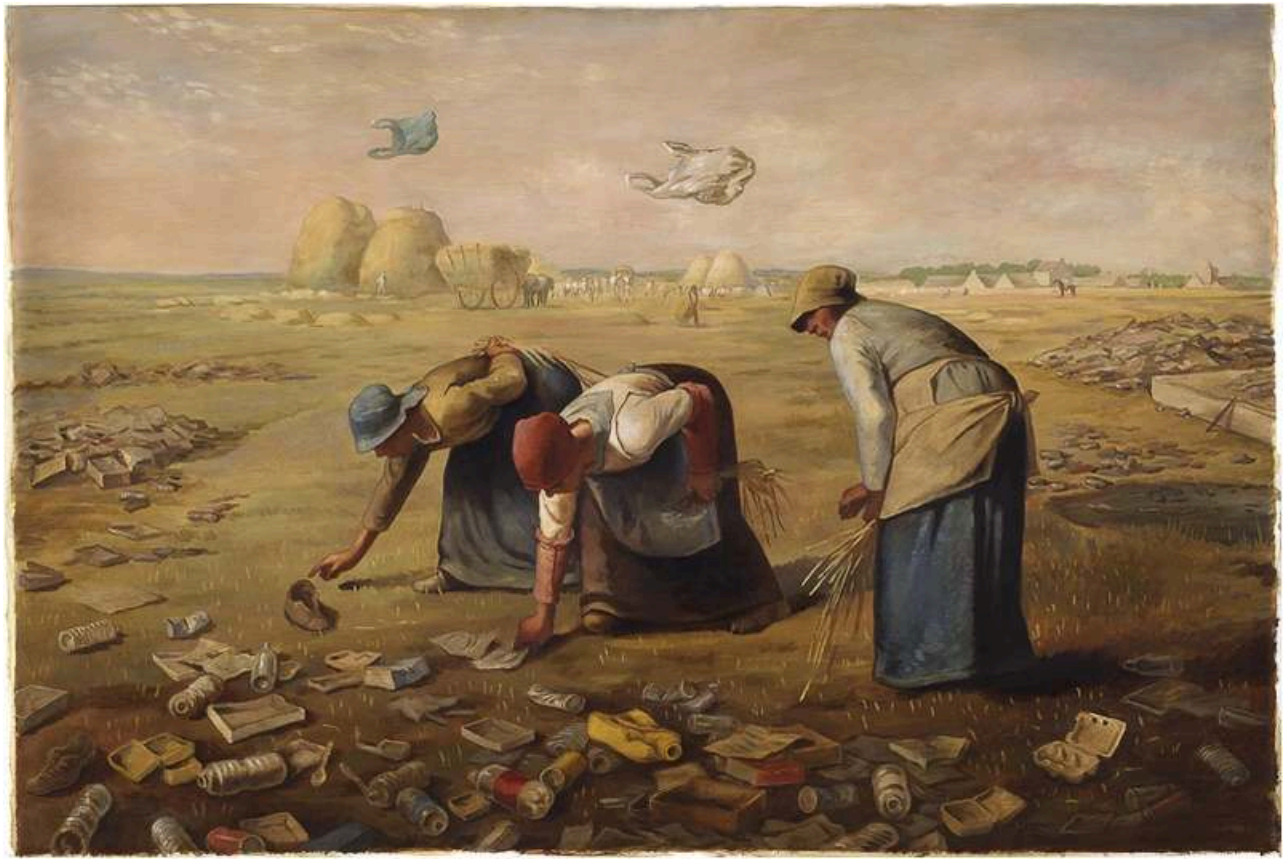

Auteur : (c) E. Leclerc et Nettoyons la nature. Illustrateur : M. Hargreaves, Agence Marie Bastille.

Collecter : XIV siècle. Dérivé de collecte. Recueillir, rassembler, faire la collecte de quelque chose.

(Synonymes : cueillir, ramasser, glaner, récupérer, prélever). 
Source : Dictionnaire de l'Académie Française, $9^{e}$ édition, 2019.

1 En 2017, le graphiste britannique, Martin Hargreaves, spécialisé dans le pastiche de tableaux emblématiques, détourne le chef-d'œuvre Des Glaneuses de Jean-François Millet (1857). Cette fameuse peinture représente trois paysannes qui ramassent les épis restant d'une récolte de blé, que l'on aperçoit à l'arrière-plan chargée sur des charrettes. Dans le détournement de M. Hargreaves, les paysannes ramassent... des déchets. Reprise par une grande chaîne de distribution française dans le cadre d'une campagne publicitaire sur l'environnement, cette composition a été affichée dans les stations du métro parisien et diffusée dans la presse et sur Internet. La représentation est doublement réussie, tant dans la communication du message de sensibilisation souhaitée par le commanditaire, que dans le rapprochement de deux activités a priori dissemblables: le glanage des restes agricoles et la récupération des déchets qui consistent dans les deux cas à collecter ce qui a été délaissé par d'autres, a priori sans valeur. Des épis de blés oubliés aux déchets, les glaneuses ramassent toujours et continuent à récupérer les restes.

2 Du latin colligere (collecter, cueillir, ramasser, recueillir, rassembler), le terme de «collecte» se veut ici une dénomination englobante, qui qualifie les processus de ramassage de matières valorisables, disponibles dans des environnements situés. Le produit de la collecte est donc une ressource, a priori renouvelable. Il ne s'agit pas d'un produit issu du travail opéré sur des matières premières : il est présent dans la nature ou dans l'environnement. C'est un produit que le cueilleur trouve, dont il connaît les lieux de concentration, dont il peut même faciliter la régénération, mais dans la collecte duquel intervient un facteur chance et une part d'incertitude.

Prélever des ressources dans son environnement plutôt que les produire est souvent perçu comme une survivance, une activité associée à la pauvreté ou au primitivisme des théories évolutionnistes; mais comme avertit Anna L. Tsing «ce serait une erreur de voir le commerce des [champignons] Matsutakes comme une survivance du passé ; c'est l'erreur faite par ceux que le progrès rend aveugle»(Tsing, 2017, p. 32). La pratique perdure au sein de nos sociétés capitalistes; elle se développe même, que ce soit pour survivre à la pauvreté, par le choix d'un mode de vie connecté à son environnement, pour répondre aux besoins grandissants en plantes sauvages des industries cosmétiques ou pour alimenter le marché florissant du recyclage. L'activité du ramassage contribue en effet à des économies dynamiques car elle est à la base de chaînes de production, guidées par de complexes réseaux relationnels et insérées dans des flux multi-scalaires insoupçonnés.

Ce dossier d'EchoGéo propose de considérer cette pratique de la « collecte », des plantes comme des détritus, dans différents espaces. De la collecte des grains de blés restant après la récolte à celle des déchets, de la cueillette des plantes médicinales au glanage urbain, la mise en perspective de plusieurs formes de prélèvement éclaire l'ambivalence d'une pratique multiforme, à la fois héritière d'une histoire longue de l'humanité où le fourrageage a pu être considéré comme "la première adaptation à succès de l'humanité » (Lee et al., 1999) et terriblement contemporaine par son inscription dans une "économie péri-capitaliste» (Tsing, 2017). Ancrée dans une certaine «tradition ", la collecte porte en elle les évolutions de notre monde. Ce dossier invite à réfléchir aux formes qu'elle prend aujourd'hui, à la manière dont elle s'opère et 
s'intègre dans l'économie mondialisée et ce qu'elle signifie dans un monde qui l'a toujours connue... mais la connaît fort peu.

\section{Un renouveau des recherches sur les collectes}

Quel que soit l'environnement, que l'on soit sur une décharge ou en forêt, les collecteurs, organisés ou pas, prélèvent des ressources. Larissa Adler Lomnitz décrivait déjà dans les années 1970 les récupérateurs de déchets de Mexico comme des «chasseurs-cueilleurs dans la jungle urbaine» (1975). Les produits de la collecte alimentent des filières domestiques, commerciales voire industrielles. L'organisation de ces filières et des relations entre les différents acteurs qui les composent a été l'objet d'un nombre croissant de recherches qui mettent en lumière les processus d'adaptation des collecteurs et leurs modalités d'insertion à l'économie globale. D'un côté, les travaux sur les déchets urbains se multiplient (Jeanjean, 2016; Florin et Cirelli, 2015). Ils explorent les mécanismes de valorisation des déchets, les remises en question des rapports de pouvoir au sein des filières et les enjeux d'un encadrement de ces activités souvent informelles, en particulier au Sud (Bercegol, Cavé et Nguyen, 2017). Considéré comme un bien économique, le déchet se caractérise à ce titre par une rivalité dont les conflits d'appropriation sont les symptômes (Cavé, 2013 ; Gidwani, 2012). Dans des villes marquées par de fortes inégalités, l'économie de la collecte et les filières protoindustrielles de transformation associées restent de grandes pourvoyeuses d'emplois (Florin, 2015). De nombreuses recherches prônent ainsi une meilleure régulation de cette économie afin de permettre la mise en place progressive de normes sociales et environnementales, les chaînes de recyclage étant peu contrôlées (Le Meur, 2016). De l'autre côté, les travaux portant sur la cueillette commerciale de plantes insistent sur les transformations de l'activité en lien avec une demande croissante et sur les enjeux de qualité des matières premières végétales (Springer, 2015; Blaikie, 2014), sur le déploiement de réseaux transfrontaliers, souvent difficiles à contrôler (Saxer, 2013) ou sur les changements de perception du produit cueilli le long de la filière (Mercan, 2012 ; Garreta, 2007). Des recherches émergentes s'attachent à décrire les pratiques et l'univers des cueilleurs (Pinton et al., 2015 ; Julliand, 2008). Le magistral ouvrage d'A. L. Tsing éclaire également les multiples enchevêtrements d'échelles qui caractérisent les filières de ces produits collectés, à travers l'exemple du champignon matsutake, cueilli en Oregon par des travailleurs précaires et revendu à prix d'or dans les épiceries japonaises de luxe (Tsing, 2017). C'est cette imbrication des échelles et des espaces qui est d'ailleurs discutée dans la rubrique Sur l'écrit de ce numéro à travers l'interview de Mathieu Quet, auteur d'une enquête fascinante sur le régime logistique des flux de médicaments. Dans ces deux études, aussi passionnantes l'une que l'autre, les filières se caractérisent par l'imbrication du légal et de l'illégal, du formel et de l'invisible. De même, alors que la collecte des déchets ou des plantes sont à la base de filières génératrices de valeur, on ne peut que constater une relégation de la pratique, cantonnée à la marginalité. Des deux côtés de la comparaison entre cueilleurs de plantes et ramasseurs de déchets se trouvent des groupes sociaux majoritairement pauvres, dont les métiers, peu reconnus par les gouvernements, sont qualifiés d'informels. 


\section{"Les cueillettes sont des stigmates de pauvreté » (Larrère, 2012, p. 40) : de la précarité subie à la marginalité choisie}

6 Le tableau des Glaneuses de Jean-François Millet, dont nous parlions au début de ce texte, fait partie de la prestigieuse collection du Musée d'Orsay et est désormais considéré comme un chef-d'œuvre inestimable. Pourtant lorsqu'en 1857 le peintre présenta son tableau au "Salon ", l'accueil des critiques fut très mitigé. Les "trois glaneuses ont des prétentions gigantesques : elles posent comme les trois Parques du paupérisme " écrivait l'essayiste Paul de Saint-Victor (1825-1881). La composition classique de la peinture, quasi-religieuse alors qu'il s'agit d'une scène de genre paysanne, était une insulte aux bonnes convenances. Le glanage était alors considéré comme une activité de survie des populations pauvres, une pratique issue d'un droit coutumier apparu au Moyen-Âge, qui consistait à permettre à ceux qui le voulaient de ramasser sur le sol ce qui restait après la récolte, offrant ainsi un complément d'alimentation gratuit. Souvent confondu avec le maraudage (le vol de récoltes non détachées du sol), hier comme aujourd'hui, l'acte de se baisser pour ramasser ce qui a été délaissé par d'autres souffre généralement d'une image négative dans nos sociétés industrialisées. Le terme de glanage est en tout cas encore utilisé de nos jours, et plus seulement dans les campagnes, mais aussi en ville, pour désigner la récupération des restes laissés dans les poubelles, une pratique dont se réclame par exemple les biffins parisiens de l'association Amélior ou les glaneurs merveilleusement mis en lumière par Agnès Varda dans son documentaire Les glaneurs et la glaneuse (2000). C'est d'ailleurs du glanage de pommes dans les vergers oubliés de Bourgogne que Mathias Faurie, artisan cidrier, tire la majeure partie de sa production, comme il nous l'explique dans un entretien pour la rubrique Sur le Métier.

7 Travailleurs pauvres, peu considérés, les collecteurs ne composent pour autant pas un groupe homogène et leur professionnalisation tend encore à diversifier leur profil. Dans ce dossier, les articles proposés ne traitent pas de populations autochtones et de leurs activités de cueillette : la collecte semble ici définitivement extraite du carcan des préjugés qui la renvoyaient à une forme d'économie primitive ou à une activité « ethnique ». Seule exception: l'article de Lena Springer qui décrit le processus de reconnaissance des connaissances et des pratiques des cueilleurs de plantes dans les montagnes du nord-ouest de la Chine, mais si l'activité de cueillette s'inscrit bien dans une identité régionale culturelle forte, le travail de valorisation mené passe par la construction de catégories plus élaborées qu'une simple spécificité ethnique. Alors qu'en Inde du Sud la collecte des produits forestiers non ligneux est réservée aux membres des Tribus recensées dans la Constitution, les cueilleurs de plantes médicinales, y compris en forêt, sont dans les faits issus de différentes populations rurales (Dejouhanet, 2014). De même, si l'on suppose que la récupération des déchets incombe dans les villes indiennes aux Intouchables, elle occupe en réalité une variété beaucoup plus large de groupes sociaux (Gill, 2009). Les collecteurs échappent dans les faits à toute essentialisation.

8 La demande de plus en plus forte en plantes incite également de nouvelles populations à s'investir dans cette activité et à s'inscrire dans des réseaux de commercialisation. Ainsi, au Québec Sabrina Doyon distingue deux profils de cueilleurs en fonction de leur investissement dans l'activité : les cueilleurs dont l'activité de cueillette est l'activité 
principale et ceux dont la cueillette est un revenu complémentaire. Les premiers ont des savoirs et savoir-faire et des ressources financières qui leur permettent de prélever des produits forestiers non ligneux sur de vastes territoires, et ils exercent ce métier par envie de rester au pays et par passion. Les cueilleurs « de complément » sont, eux, des collecteurs irréguliers, approvisionnant les petites entreprises ou, en rejoignant un camp de cueilleurs, fournissant de gros volumes de matières premières aux industries transformatrices. Leur activité de cueillette n'est pas nécessairement déclarée et ils sont souvent en situation économique précaire, puisqu'ils cumulent différentes sources de revenu. Il s'agit d'une population instable dans sa pratique : ils choisissent leurs débouchés en fonction des opportunités, peuvent se désister en fonction des années, des saisons ou des sommes proposées.

9 C'est bien de cette distinction, qu'il faudrait sans doute nuancer, entre des collecteurs " professionnels » et des "non professionnels », qu'est parti le projet de formalisation de la cueillette française porté par l'AFC (Association française des professionnels de la cueillette) que nous présentent Claire Julliand et al. Les collecteurs " professionnels " auraient conscience du besoin d'adopter des pratiques de cueillette durables et respectueuses de l'environnement, tandis que les autres, attirés dans cette activité par son revenu potentiel, cueilleraient sans se préoccuper d'une gestion à long ou même à moyen terme de la ressource. La formalisation du métier de cueilleur s'intègre alors dans une opposition entre gestion raisonnée des ressources et prédation, les mauvaises pratiques servant à définir ce que doit être une collecte respectueuse de son milieu. Dans un sens, on retrouve une catégorisation semblable entre «bons récupérateurs » et "mauvais récupérateurs» parmi les ferraillous de région parisienne décrits par Bénédicte Florin et Pascal Garret : la précarité de certains collecteurs les assigne en effet à être mal considérés par les autres, quelles que soient leurs pratiques. En filigrane, ces représentations interrogent l'ambivalence dans l'activité de collecte entre une marginalité choisie et une précarité subie.

\section{"Les res derelictae sont devenues une ressource commune » (Glémain, 2013, p.157) : des ressources à sécuriser}

10 La précarité des statuts rend la question de l'accès à la collecte particulièrement importante. Les ressources sont disponibles dans l'environnement, selon soit un système de biens de club lorsque certaines espèces sont réservées (carte de collecte de champignons, droits associés sur certains territoires à une catégorie particulière de cueilleurs, délégation de la collecte des déchets à un seul opérateur privé sur le territoire), soit un système de libre accès où les premiers arrivés sont les premiers servis. Les rationalités accompagnant la collecte peuvent alors être fortement conflictuelles dans la définition des droits sur les ressources, dans la compétition spatiale pour leur collecte et dans l'inégale redistribution des richesses qui découlent de leur vente et de leur transformation, surtout quand elles sont associées à une faible régulation étatique. En ville, le déchet, res derelictae, est théoriquement considéré comme res nullius (tout comme les plantes rudérales) : à partir du moment où son propriétaire décide de s'en débarrasser, n'importe qui peut se l'approprier, il entre dans le régime des biens en accès libre. Dans les faits, les personnes qui fouillent dans les poubelles pour y récupérer des aliments ou des biens sont souvent considérées 
comme outrepassant les droits de l'ex-propriétaire des déchets comme le montre bien l'expérience de Jeanne Guien, freegan parisienne. De même, B. Florin et P. Garret nous donnent à suivre les ferraillous franciliens qui souffrent d'une réputation sulfureuse, comme parmi eux les Roms souvent qualifiés de voleurs. La compétition pour l'accès à la ressource se fait alors entre les récupérateurs et les anciens propriétaires du déchet, entre les entreprises de ramassage qui bénéficient d'une légitimité sociale et symbolique pour s'occuper de cette récupération et les récupérateurs informels qui alimentent généralement des centres de tri ou des ferrailleurs, et entre les récupérateurs eux-mêmes qui déploient des stratégies pour être les premiers sur le site de dépôt des déchets.

Tout un système de réglementation se met ainsi peu à peu en place pour encadrer la cueillette des plantes sauvages, visant d'une part à protéger les espèces vulnérables et d'autre part à préserver certains espaces d'une exploitation des ressources. Des préconisations de techniques de cueillette et de gestion de la ressource ont été proposées dans le passé, mais elles ont été peu suivies. Ce sont désormais des chartes construites selon des méthodes participatives - par exemple, la charte nationale de cueillette de l'AFC ${ }^{1}$, celle d'éco-cueillette du Morvan et de la Bourgogne ${ }^{2}$ - qui visent à contrôler et structurer les pratiques de cueillette. Dans le contexte de compétition pour les ressources, la formalisation des activités de ramassage par les récupérateurs est alors pour eux un moyen d'exister face à des entreprises justifiant de contrats avec les municipalités par exemple, comme le décrivent Mélanie Rateau et Luisa Tovar pour les collecteurs latino-américains. Mais cette formalisation des pratiques, du métier, des réseaux de commercialisation semble engendrer une double tension, ce qui explique sans doute les avis partagés des cueilleurs au Québec (S. Doyon) ou en France (c. Julliand et al.) sur ce processus. D'un côté la formalisation permet d'entrer dans un système de normes et de bénéficier d'une reconnaissance officielle de la profession, de l'autre elle limite la liberté qui caractérise le métier et ses pratiques (les champignons sont présentés comme des "trophées de la liberté " par A.L. Sting). La formalisation transforme la pratique de collecte et le statut des collecteurs, alors que ces groupes peu organisés ont un capital social peu développé, les reléguant parfois à une nouvelle marge comme l'expliquent Mathieu Durand et Fabio de Oliveira Neves à partir d'expériences de formalisation en Amérique Latine. Plutôt qu'une formalisation, c'est avant tout une reconnaissance de leur existence que ces travailleurs réclament. C'est cette perspective d'humanisation d'un droit à la collecte, qui est développée dans la rubrique Sur l'Image avec la présentation de quelques photographies extraites de l'exposition « La mise en image du rebus » par C. Cirelli et al.

\section{"La précarité est la condition de notre temps » (Sting, 2017, p. 55) : de la collecte comme résistance}

12 Raphaël Larrère (2012) analysait la cueillette des champignons en Margeride dans les années 1970-80 selon trois prismes : comme des ruses pour résister à la paupérisation, comme une réappropriation symbolique du territoire, ou comme un des rares loisirs des populations rurales isolées. Dans le cas des cueilleurs en Margeride, la collecte se faisait résistance contre une précarisation des conditions de vie des paysans, dépossédés d'une partie de leurs terres rachetées et progressivement reboisées : si les notables avaient pris la terre, les paysans gardaient tout de même les champignons 
(Larrère, 2012). La revendication symbolique associée à la cueillette et au bénéfice des ressources du territoire de vie se retrouve dans de multiples situations : au Québec où le développement des activités de cueillette s'inscrit dans une recherche de maintien des jeunes "en région" et de développement régional (S. Doyon), en Chine où la valorisation des savoirs des cueilleurs s'accompagne d'un réinvestissement dans les traditions locales et la religion daoïste, en opposition avec les campagnes passées d'homogénéisation culturelle (L. Springer), ou en région parisienne où les Roms relogés pratiquent quand même la récupération même si leur relogement était conditionné à l'arrêt de cette activité (B. Florin et P. Garret). La collecte apparait alors comme le symbole d'une résistance dans un monde précarisé (Tsing, 2017).

Il est difficile de contrôler l'informel, donc les politiques publiques encouragent l'organisation formelle des groupes de collecteurs, de leurs pratiques et des activités liées à la collecte. Christer Norström (2003) définissait cependant le cueilleur par le concept d'autonomie : autonomie collective et individuelle. La formalisation est-elle alors une normalisation qui viendrait brider la richesse de cette pratique résistante ou est-elle la possibilité de préserver une liberté d'exercice de ce métier, un droit à la marge, dans un monde qui se normalise ? Quelle place est laissée pour la collecte dans un monde dans lequel le contrôle sur les activités se veut de plus en plus serré et les normes plus étroites ? Y a-t-il encore de la place pour les marges ? Les aires protégées, la mise en défens des forêts à champignons pour les réserver aux détenteurs d'une carte (Barreau, 2014; Tsing, 2017), l'étalement urbain, la déforestation au sud, l'aménagement sécuritaire des villes, etc. semblent repousser toujours plus loin l'espace de cette liberté qu'est la marginalité, le rendant plus étroit. Si A. L. Tsing affirme que la durabilité des sociétés est à chercher dans les marges, là où la précarité invite à réinventer les manières de faire monde, elle propose aussi de revisiter la définition des marges : au cœur des espaces industrialisés, exploités, là où le Progrès pense n'avoir rien laissé d'exploitable. À la lumière de cette réflexion, les cueillettes urbaines prennent tout leur sens. Les habitants de Cayenne présentés par MarcAlexandre Tareau et al., sont nouvellement arrivés des périphéries rurales de la région et amènent leurs habitudes de soin par les plantes avec eux ; pour les faire perdurer, ils cherchent alors les plantes qui poussent dans l'environnement urbain. S'ils ne peuvent évidemment pas trouver toutes les espèces de leur pharmacopée, ils mettent en place des stratégies de cueillette au sein de l'espace urbain et de ses périphéries pour trouver les espèces principales, souvent rudérales. Ce sont donc les dents creuses, les friches urbaines et les arbres dépassant des jardins qui sont exploités, dans un espace de cueillette tracé à travers la ville en un archipel de lieux de cueillette.

Cette appropriation par la cueillette de ressources urbaines est à mettre en parallèle, comme l'avait fait avant nous Agnès Varda (2000), avec les pratiques alimentaires urbaines de glanage et grappillage. Désormais émergent aussi de nouvelles formes de collecte urbaines : la cueillette de plantes alimentaires qui se développe dans les villes américaines et européennes, et le freeganisme qui consiste à fouiller les poubelles pour se nourrir dans une démarche militante et anticapitaliste. L'adaptation au contexte urbain des cueilleurs de plantes guyanais et la recherche de nouvelles ressources alimentaires en environnement urbain par les cueilleurs new-yorkais, dont nous parle Flaminia Paddeu, sont des cueillettes engagées, dans lesquelles s'affirme une autre manière de voir le monde et une volonté d'exister par soi-même. Dans la contestation du gaspillage alimentaire, le freegan, incarné ici par J.Guien, parasite le système 
économique ; il trouve sa survie dans ce gaspillage tout en le dénonçant, il se nourrit lui aussi dans les « ruines du capitalisme ». Le cueilleur de plantes urbaines, lui, réinvente la ville en valorisant les espaces de nature non aménagés, les espaces qui échappent au contrôle des urbanistes et qui n'ont aucune valeur à leurs yeux. Les cueilleurs de l'Oregon d'A.L. Sting développent leurs activités dans des environnements dégradés, où de nouvelles collaborations écologiques ont permis la pousse du Matsutake, champignon dont la valeur sur le marché mondial est forte. De même, les ramasseurs de déchets, produits et survivants d'un monde du périssable, à première vue semblant marginaux et hors du système, sont pourtant bien ancrés dans un système capitaliste qui fait par exemple du recyclage du plastique un phénomène économique mondial. Ces manières émergentes de pratiquer la collecte, au cœur des ruines, montrent s'il le fallait que la cueillette est une manière de dépasser et de valoriser un environnement même dégradé, de survivre et de proposer une autre manière de penser le monde ${ }^{3}$.

\section{Conclusion}

«Des manières de faire-monde émergent des activités pratiques déployées pour se tenir en vie et ne cessent d'altérer la planète. Pour les apercevoir, dans l'ombre de l'« anthropo- ", nous devons changer de point de vue. Alors qu'ils étaient courants à l'époque préindustrielle, de nombreux moyens d'existence, de la cueillette au vol, persistent encore aujourd'hui. Et de nouveaux, encore, font leur apparition (y compris la cueillette commerciale des champignons). Mais ces manières de vivre et de faire, parce qu'elles sont considérées comme n'appartenant pas à la marche du progrès, sont négligées. Ces moyens d'existence, pourtant, fabriquent aussi le monde et, surtout, nous montrent comment regarder autour de nous, plutôt qu'en avant. » (Tsing, 2017, p. 58).

16 Ce dossier thématique d'EchoGéo propose de décentrer le regard comme le suggère A.L. Sting pour percevoir ces autres manières de faire-monde, ces multiples projets de fabriques de mondes qui se chevauchent, ignorés par une approche linéaire du développement et du progrès. La collecte devient alors une lunette, un prisme pour regarder les évolutions sociales, économiques et culturelles de notre temps. Le grand écart entre cueillette de plante et récupération des déchets n'est qu'apparent : cette mise en perspective s'avère une passerelle qui invite à réfléchir au sens de la collecte, à la cueillette aujourd'hui. En comparant des espaces et systèmes de collecte a priori très différenciés, des similarités intéressantes émergent et permettent de mieux comprendre la signification de l'acte même de la collecte.

17 Regardons encore une dernière fois nos trois glaneuses. Courbées vers le sol, chacune d'entre elles représente l'une des phases de ce mouvement ancestral de la collecte. Alors que celle de droite observe et recherche, celle de gauche a trouvé, le doigt tendu vers l'épi/déchet, et celle du centre, dont la main touche le sol, en ramasse un. Ce qui en fait un chef-d'œuvre n'est pas seulement le bouleversement de la hiérarchie des genres qui a pu choquer les critiques les moins perspicaces, c'est aussi et surtout la capacité du peintre à pouvoir conférer à ces glaneuses une dignité, "une valeur d'emblème dénuée de tout misérabilisme " (description du Musée d'Orsay). Sans avoir d'aussi hautes prétentions, c'est bien dans cette démarche de mise en lumière que ce dossier d'EchoGéo a été conçu : donner à voir la collecte par ce qu'elle est et permet de 
faire, analyser ce qu'elle révèle, au-delà des idées préconçues, comme un acte fort reliant des espaces entre eux et contribuant à faire le monde.

\section{BIBLIOGRAPHIE}

Blaikie C., 2014. Making medicine: Pharmacy, exchange and the production of Sowa Rigpa in Ladakh. Thèse de doctorat en anthropologie, Université de Kent.

Barreau G., 2014. Boletus : fructus, usus et abusus. Cahiers d'Etudes Forêt, Environnement et Société, $\mathrm{n}^{\circ} 24$ ("Les usages non ligneux de la forêt"), p. 80-86.

De Bercegol R., Cavé J., Nguyen Thai Huyen A., 2017. Waste Municipal Service and Informal Recycling Sector in Fast-Growing Asian Cities: Co-Existence, Opposition or Integration? Resources, vol. 6, n 4, 70. https://doi.org/10.3390/resources6040070

Cavé, J. 2014. Who owns urban waste? Appropriation conflicts in emerging countries. Waste Management and Research, $\mathrm{n}^{\circ} 32$, p. 813-821.

Dejouhanet L., 2014. Secteur informel et réseaux de commercialisation des plantes médicinales au Kérala (Inde). Économie rurale, $n^{\circ} 343$, p. 53-70.

Florin B., 2015. Les chiffonniers du Caire. Soutiers de la ville ou businessmen des ordures? Ethnologie française, $\mathrm{n}^{\circ} 153$, p. 487-498.

Florin B., Cirelli C. (dir.), 2015. Sociétés Urbaines et déchets. PUFR, 452 p.

Glémain P., 2013 Economie des res derelictae et gestion solidaire des déchets. Les écocycleries, des entreprises d'appropriateurs solidaires. Management \& Avenir, $\mathrm{n}^{\circ}$ 65, p. 154-168. https:// www.cairn.info/revue-management-et-avenir-2013-7-page-154.htm

Jeanjean A., 2016. Peindre la voix, écrire le déchet. Techniques \& Culture [En ligne], n 65-66 ("Réparer le monde : excès, reste et innovation"), p. 310-321. http://journals.openedition.org/tc/ 8075

Le Meur M., 2016. Sous la montagne de plastique, une mine d'or ? Le mythe du recyclage à l'épreuve d'une filière vietnamienne. Techniques \& Culture [En ligne], n 65-66 ("Réparer le monde : excès, reste et innovation"), p. 202-205. http://journals.openedition.org/tc/7951

Lee R.B., Daly R., Heywood Daly R., 1999. The Cambridge Encylopedia of Hunters and Gatherers. Cambridge University Press, $511 \mathrm{p}$.

Lomnitz L.A., 1975. Cómo sobreviven los marginados. Mexico, Siglo XXI Ed., 229 p.

Garreta R., 2007, Des simples à l'essentiel. Toulouse, Presses Universitaires du Mirail, 368 p.

Julliand C., 2008. Itinéraires de cueillette : cheminement au cœur et aux marges d'une pratique sociale, économique et symbolique. In Hallé F., Aux origines des plantes, Paris, Fayard, p. 502-529.

Larrère R., 2012. Cueillettes, menues glanes et pauvreté. In Dalla Bernardina S., L'appel du sauvage. Refaire le monde dans les bois. Rennes, Presses Universitaires de Rennes, p. 33-41.

Mercan A., 2012. La route du cordyceps. Autrepart, n 63, p. 89-105. https://www.cairn.info/ revue-autrepart-2012-4-page-89.htm 
Pinton F., Julliand C. et Lescure J.-P., 2015. Le producteur-cueilleur, un acteur de l'interstice? Anthropology of food [En ligne], S11. http://journals.openedition.org/aof/7902

Saxer M., 2013. Manufacturing Tibetan Medicine. The Creation of an Industry and the Moral Economy of Tibetanness. New York, Berghahn, 304 p.

Springer L., 2015. Collectors, Producers, and Circulators of Tibetan and Chinese Medicines in Sichuan Province. Asian Medicine, vol. 10, n 1-2, p. 177-220. doi:10.1163/15734218-12341357

Sting A. Lowenhaupt, 2017. Le champignon de la fin du monde. Sur la possibilité de vivre dans les ruines du capitalisme. Paris, La Découverte, 416 p. [Traduction de l'ouvrage publié en 2015 The Mushroom at the End of the World: On the Possibility of Life in Capitalist Ruins].

Varda A., 2000. Film documentaire. Les glaneurs et la glaneuse. Ciné Tamaris, $82 \mathrm{mn}$.

\section{NOTES}

1. http://www.cueillettes-pro.org/L-etude-Charte.html, consulté le 31 mars 2019.

2. http://www.parcdumorvan.org/fic_bdd/pdf_fr_fichier/1149613688_ECO_CUEILLETTE.pdf, consulté le 31 mars 2019.

3. L'approche sensible inconditionnellement liée à la cueillette des plantes sauvages, est peu développée ici. Elle pourrait pourtant être pertinemment rapprochée de la philosophie et la cosmologie développées autour de la récupération, des déchets ou des aliments jetés.

\section{INDEX}

Thèmes : Sur le Champ - Sur le Terrain

\section{AUTEURS}

\section{LUCIE DEJOUHANET}

Lucie Dejouhanet, lucie.dejouhanet@orange.fr, is a Senior Lecturer at University of Antilles and a member of AIHP-GEODE laboratory, EA 929. She recently published :

- Dejouhanet L., 2014. Supply of Medicinal Raw Materials. The Achilles Heel of Today's Manufacturing Sector for Ayurvedic Drugs in Kerala. Asian Medicine, vol. 7, n 3, p. 206-235.

- Dejouhanet L., 2014. Secteur informel et réseaux de commercialisation des plantes médicinales au Kérala (Inde). Économie rurale, $\mathrm{n}^{\circ} 343$, p. 53-70.

- Dejouhanet L., 2017. Tourism in the mountains of Central Kerala (South India): at the Crossroads of attitudes toward forest populations. Journal of Alpine Research, vol. 105, $\mathrm{n}^{\circ} 3$ / Le tourisme dans les montagnes du centre du Kérala (Inde du Sud) : à la croisée des regards posés sur les populations forestières. Journal de Géographie Alpine, vol. 105, nº 3. 


\section{RÉMI DE BERCEGOL}

Rémi de Bercegol, remi.debercegol@cnrs.fr, est chargé de recherche au Centre National de la Recherche Scientifique, membre de l'UMR PRODIG (Paris). Il a co-organisé à Delhi l'exposition The City of Waste, version anglaise de l'expo dont sont extraites les photos. Il a publié récemment :

- de Bercegol R., 2019 [1ère ed. 2010]. Small Towns and Decentralisation in India. Rawat Publications [1ère ed. Springer], $246 \mathrm{p}$.

- de Bercegol R., Gowda S., 2018. Waste and Energy Nexus: Rethinking the modernisation of waste services in Delhi. Urban Studies, Special Issue on 'Urban Nexus', June 2018.

- de Bercegol R,, 2018. Les enjeux de la valorisation des déchets à Delhi. In Jaglin S, Debout L., Salenson I., (dir.), Du rebut à la ressource, Valorisation des déchets dans les villes du sud. Paris, AFD, p. 31-49. 